\title{
Towards a sociological understanding of sexting as a social practice: a case study of university undergraduate men
}

Steven Roberts, Monash University

Signe Ravn, University of Melbourne

This is the accepted version of the article, ie the final version before layout and typesetting. Some minor changes may have occured in the proof stages. Figures are omitted in this version.

The full reference is: Roberts, S. \& Ravn, S. (2020): “Towards a sociological understanding of sexting as a social practice: a case study of university undergraduate men”. Sociology, vol. 54(2), pp. 258-274.

https://doi.org/10.1177/0038038519864239

\begin{abstract}
This paper makes the case for understanding young people's engagement with 'sexting' as a social practice. Moving away from the dominant focus on teenagers and (sexual) risk and instead approaching sexting as an 'everyday' practice sheds light on how sexting is perceived and situated as a normalised part of contemporary youth culture. Drawing on 10 focus groups with 37 undergraduate men in Melbourne, Australia, our data reveals young men's significant emphasis on consent, mutuality and respect, marking out 'appropriate sexting' practices as distinct from harassment or image-based abuse. Nonetheless, the centrality of a transactional approach to sexting questions those seemingly positive dispositions. Social practice theory permits sophisticated understanding of these nuances, seeing them as bound up and produced in correspondence with the broader meanings, embodied skills and material artefacts that are associated with sexting.
\end{abstract}

Keywords: youth; youth culture; sexting; sexuality; practice theory; qualitative methods 


\section{Introduction}

Concerns around sexting - i.e. the 'creation and sharing of sexual images or text messages via mobile phones or internet applications' (Hassinoff, 2014: 449) - appear as one of the latest 'moral panics' (Cohen, 1972) over young people's doings. Young people are depicted as naïve and ignorant in sending suggestive or nude images, with no awareness of potential negative repercussions in the present or the future (Mitchell et al., 2012), as well as being careless and potentially criminal in the ways in which they share and store such images (Ringrose and Harvey, 2015; Salter, 2016). In popular discourse sexting is often grouped together with image-based abuse ${ }^{i}$ (Henry et al., 2017), as premised on the same underlying logic of risk and female victimisation, and the best advice given from schools, authorities and public campaigns seems to be: 'abstain' (Albury et al., 2013). Uniting these concerns is a lack of engagement with young people's own understandings of the norms around sexting and the role of sexting in their lives.

This paper contributes to the sociological literature by delivering a more nuanced understanding of how sexting is perceived and situated in young people's lives. Our primary contribution is conceptual and pertains to young people's practice of sexting, rather than being about men and masculinity per se. That said, our paper is informed by wider debates about men, masculinity and sexuality. Our arguments resonate with recent discussions emphasising 'significant social change related to sexuality has occurred over the past 30 years' (McCormack and Wignall, 2017: 975). This attention to social change has, for example, highlighted the relative normalisation of hook up culture among university students (Armstrong et al., 2010). Our approach is also commensurate with emerging work on young men's sexuality that moves away from a longstanding focus on sexual harms (e.g. Salter, 2016) to exploring their own understandings, experiences and motivations for their sexual practices and situating this in their broader everyday lives. For instance, in their investigation of young men's pornography use, McCormack and Wignall (2017) make a compelling case for understanding porn consumption as a casual leisure activity. Similarly, troubling the automatic acceptance of the sexual risk/harm paradigm, Waling and Pym (2019) highlight how the conflation of solicited and unsolicited dick pics renders invisible women's sexual agency, reduces the male body to being 'shameful', and situates men's sexual behaviour as essentially ‘creepy' (Waling and Pym, 2019: 79-80). 
In line with this work, and taking sexting as our focus, our paper investigates the duality of sexting's cultural positioning as inherently risky versus sexting as an accepted and regular form of sexual expression. Drawing on a focus group study with 37 undergraduate men in Victoria, Australia, we take a novel conceptual approach to analysing sexting; that is, through deploying social practice theory (SPT). Using this analytical lens, we explore how sexting is understood and experienced by young men. As we demonstrate, this approach allows us to unpack how cultural meanings and norms are translated into, first, competencies and then a complex set of actual practices that our participants must learn. Our participants note that 'good' sexting demands consent and mutuality, but that this is complex given the perceived understanding of the need to engage in a stepwise escalation of sexualised content as the sexting encounter unfolds. Despite these ambiguities, we situate sexting as not inherently problematic but as an important and highly normalised part of contemporary youth cultures, distinct from both flirting and harassment. That is, we see the practice of sexting being part of the 'cultures of 'shared ideas', whose interactions take place not in physical locations such as the street, club or festival field but in the virtual spaces facilitated by the Internet' (Bennett, 2004: 163). Understanding how these 'cultures of shared ideas' combine with the virtual terrain where sexting takes place also permits us to extend SPT into new territory to fruitfully explore how social practices are learned. Where SPT often marks out co-presence of a physical other as central to learning a practice, here we explore how the mediated presence of the other is central to - and also complicates - the learning process.

\section{Young people and sexting: trends and gaps in the literature}

Existing research on sexting has mostly focused on youth, being variously concerned with prevalence (e.g. Klettke et al., 2014), motivations (e.g. Lee et al., 2015), behaviours and experiences (e.g. Albury et al., 2013; Ringrose and Harvey., 2015), and media and political representations (e.g. Hassinoff, 2014). This extant research can be divided into two broad strands: one focused on potential negative consequences of sexting, the other on the increased normalcy of the practice. While the links between sexting and problematic behaviours has had substantial focus, numerous studies in the normalcy 'camp' have critiqued the pervasive 'media risk discourse', which surrounds sexting as it applies to (especially younger) groups. This work illustrates how the risk discourse presents sexting as a gendered problem, highlighting how the 'sexting panic' (Hasinoff, 2014) is concerned with 'under-aged girls lacking vigilance in their uses of social media, and boys as predatory and over-sexed' (Ringrose \& Harvey, 2015: 206). As evidenced by Madigan and colleagues' (2018) 
systematic review - summarising studies involving in total more than 110,000 youths - these media representations are grossly misplaced. Indeed, sexting is more common as teenagers get older and this is related to engagement in sexual intercourse (Madigan et al., 2018). These findings align with Albury and colleagues' (2013: 1) point that sexting appears to be 'understood as an adult or media-generated concept that [does] not adequately reflect young people's everyday practices and experiences of creating and sharing digital images'.

Accurately depicting the prevalence of sexting is difficult. Studies among adults suffer from similar inconsistencies as those among younger populations - in both cases, and even with studies involving nationally representative probability samples, results vary dramatically, with youth sexting prevalence 'ranging from $2.5 \%$ to $24 \%$ ', and adult sexting prevalence 'as low as 30\% and [in other studies] as high as 81\%' (Kosenko et al., 2017: 142). To make sense of this vast variation between studies, Kosenko et al. cite Ringrose and colleagues (2012: 12) who note 'it is difficult to know if 'sexting' is under-reported because of social desirability factors (e.g. embarrassment on the part of respondents) or over-reported because of response biases (those who do it may be more likely to respond to surveys)'.

Anastassiou's (2017: 2237) systematic review of qualitative research on sexting notes its 'immense value', but also that such investigations 'remain scarce'. The existing qualitative work has illuminated gendered dynamics of sexting, with Salter (2016: 2723; also Salter et al., 2013) arguing that 'digital images of bodies circulate online in manner that reinforces gender inequalities'. A particular focus in this literature is the sexual 'double standard' (e.g. Albury et al., 2013; Ringrose et al., 2013). Here, boys' sexting facilitates the building of social capital, establishing a perceived veneer of 'coolness' among peers; meanwhile, girls are more likely to be subjected to risks such as coercion, sometimes resulting from 'prudeshaming' for refusing to engage in sexting, and being slut-shamed for sending sexual images or text.

The ambition in our research was to further investigate these gendered dynamics by paying closer attention to how young adult men - rather than teenage boys - engage with, understand and talk about sexting. While (young) men are often positioned as the primary problematic in this gendered practice, the voices of young boys and men are often silent in research on sexuality (cf. Harvey \& Ringrose, 2015). Moreover, moving the focus to the slightly older, young adult population - young men who are (presumably) sexually active, 
and hence have (some) experiences with intimate/romantic relations - is important as there is a relative lack of studies on sexting outside of the teenage years (McDaniel \& Drouin, 2015). While some existing studies on young adults have paid attention to risk factors (e.g. Henry et al., 2017), limited research considers how sexting looks and is perceived by older age groups; groups where we can expect this practice to play another role as their sexual lives are developing.

In sum, addressing these gaps we locate our work in calls for 'more research and theory addressing how sexual pleasure exists alongside danger and risk' (Hassinoff, 2014: 458), focusing in particular on young men's understandings of the normative meanings of sexting and their engagement in this practice. Our ambition in this paper is to argue for the usefulness of SPT as a means to further discuss the relationship between contemporary youth culture, sex and sexuality and, thus, also address the concerns and panics around contemporary youth practices.

\section{Social practice theory: a brief introduction}

Previous research has considered sexting through various theoretical and conceptual lenses. These include including communication theory (Hassinoff, 2014), problem behaviour theory (Kosenko et al., 2017), feminist analysis (Albury et al., 2013; Ringrose et al., 2013; Salter, 2016) and, more recently, sexual script theory (e.g. Symons et al., 2018; Yadlosky et al., 2017). Despite its popularity in sexuality research, script theory has its limitations for studying sexting. These include that existing cultural and sexual scripts for how to engage in sexual or romantic interactions are not automatically transferable to the digital sphere, and also that sexual script theory pays limited attention to the embodied and performative dimensions of practices (for a discussion see Jackson and Scott, 2017). In this paper we follow the 'turn to practice' (cf. Reckwitz, 2002) recently emerging in wider research on sexuality (e.g. Jackson and Scott, 2017) and argue for adapting this conceptual approach to view sexting as a distinct social practice.

Discussions of practice do emerge in the literature on sexting. Albury et al (2013: 1-2) for example note, among others, 'the practice of sending naked or semi-naked pictures' and 'respectful practices between intimate partners and within friendship networks'. Meanwhile, Ringrose and Harvey (2015) signal their analysis of 'social media images and practices', and variously discuss Facebook 'commenting practices' and 'performative practices of 
heteronormative masculinity'. However, these are more everyday uses of the term practice, and is different to and distinct from theories of practice, which foreground the everyday embeddedness of practices and their normative regulation (Halkier, 2017; Warde, 2014).

SPT corresponds with our ambitions to approach sexting as an embodied practice that is grounded in everyday life and part of local (youth) cultures. In other words, this approach involves 'understand[ing] the complexities of mundane performativity' (Halkier, 2017: 395). Considering sexting a mundane activity might seem counter-intuitive, given its 'exciting' and/or 'risky' connotations. However, both the literature (Walrave et al., 2018) and our data emphasise the normalised nature of sexting and this is what prompted our turn to theories of practice in the first place.

Social practices are constituted by meanings, materials and competencies (Shove et al., 2012), or in Reckwitz's (2002:249) elaborate definition:
'A practice... is a routinized type of behaviour which consists of several elements, interconnected to one other: forms of bodily activities, forms of mental activities, 'things' and their use, a background knowledge in the form of understanding, know-how, states of emotion and motivational knowledge'.

Practices, then, are inherently social entities that exist independently of the individual, who engages in, performs, repeats and potentially alters these practices (Maller, 2015); individuals are seen as 'carriers' or performers of practices. Social practices are not the same as individual habits (Shove et al., 2012); rather, individual actions emerge from social practices. This means that approaching sexting as a social practice involves moving away from viewing this as a matter of 'aggressive' or 'oversexed' (male) individuals making poor choices and instead see this as individual performances of a broader sexting practice that has meanings, relies on embodied skills and involves material artefacts.

The meanings that sexting as a practice carry, or the 'cultural conventions, expectations and socially shared meanings' (Maller, 2015: 58) associated with sexting, are key to understanding why young men engage in sexting practices and how such engagements should look. Within a practice-theoretical framework, meanings are not located at the individual level. Practices are both dynamic and relational, and one affordance of practice theory is that 
it directs our analytical attention in two different directions: first, to the actual performances of practices - what people go about doing; and second, to the discursive level (Swidler, 2001). Swidler (2001: 75) suggests that 'a focus on discourse then reintroduces the world of language, symbols and meanings without making them anyone-in-particular's meanings'. This is central for our methodological and analytical approach, meaning that we can view focus group discussions as participants' interpretations of broader, socially shared meanings, which also point to the normative regulations surrounding a given practice (Halkier \& Jensen, 2011). Hence, while consumption researchers will ask what 'cooking properly' or 'eating healthily' involves (Halkier \& Jensen, 2011: 106), we analyse what constitutes 'appropriate' sexting by investigating how this is continuously constructed and described in discursive practices. As Halkier (2017: 39) puts it, 'to practice something in everyday life involves normative negotiation of what one can be expected and accepted to do'. This extends into competencies; knowing how to engage in a given practice and do so skilfully. Practices rely on embodied and tacit knowledge that one must learn to appropriately engage in the practice. This process of learning the tacit norms and cultivating one's skills to engage in sexting practices in a culturally accepted way is at the crux of our analysis. And finally, practices involve 'things and their use' as the quote above suggests. Practices are underpinned by materialities, in our case for instance digital technologies, that one must appropriate and learn to use and that in turn facilitate the specific practice.

\section{Methods and data}

Our data are drawn from 10 focus group interviews conducted between October 2016 and July 2017. The sample, drawn from two large universities in Melbourne, Australia, comprised 37 undergraduate young men, aged 18-22, from various study disciplines. Most focus groups contained 3-6 participants. All participants in nine of the ten groups identified as heterosexual, while one group consisted of two gay identifying men. Most participants were Australian; however, two were American, one Chinese, one Irish and one English.

The focus groups were designed to explore our overall research question; what are young men's understandings of and experiences with sexting, by illuminating collectively constructed and accepted norms around this through group discussions. For this reason, and given the potentially sensitive topic, the focus groups were comprised of existing friendship groups. This allowed us to benefit from the already established sense of trust and the social bonds between participants to make participants feel more at ease (Grogan and Richards, 
2002). While recognising this does not reflect 'real life' talk amongst participants (Demant, 2012), the focus group discussions shed light on how participants express and co-construct their understandings of acceptable social norms around sexting (Halkier, 2017: 395) as well as the broader cultural understandings that the focus groups tap into.

The focus groups followed a discussion guide covering definitions of sexting; the relation between sexting and sex; norms around sharing sexts between friends; experiences with sexting with potential/current partners; perceived gendered differences, and cultural representations of sexting and risk. Discussions were underpinned by using visual stimulus materials (Barbour, 2007; Törrönen, 2002) consisting of examples of what might be seen as sexting, sourced from Google. ${ }^{\text {ii }}$ These covered comedic, aggressive and ambiguous examples and were introduced to discuss what is seen to constitute sexting.

Each focus group took place around a table in university offices and were moderated by the male author. Pini (2005: 204) suggests that 'it is the subject of the research, not just the identities of the researcher and researched, that will shape an interview'. The lively discussions in the groups may be a result of a perceived smaller social distance between participants and the male moderator, who, while older than participants, is familiar with social media and youth (popular) culture and in that sense create an informal atmosphere in the groups.

The focus groups lasted up to 2 hours, averaging $1 \mathrm{hr} 35 \mathrm{mins}$, and were audio recorded and transcribed verbatim. The men received a $\$ 30$ retail voucher for their participation. Names and other identifying characteristics such as study programme have been anonymised. We followed conventional ethical guidelines for social science research such as seeking informed consent and ensuring a positive tone in the focus groups. [X] University granted ethics clearance (NUMBER).

Reading through the rich transcripts with our research question in mind, it became clear that the question for most participants was not whether to engage in sexting or not, but rather how sexting is and should be carried out, with whom, when etc. This initial engagement made us turn to SPT as an analytical frame. Following an abductive approach (Meyer \& Lunnay, 2013) we moved back and forth between data and theory, here SPT. Hence, we first developed three mastercodes pertaining to the 'what', 'how' and 'why' of sexting, with 
subcodes covering definitions and understandings of what constitutes 'appropriate' and 'inappropriate' sexting including consent and the implications and meaning of 'crossing the line'. To move beyond this initial layer of 'understandings' we cross coded these themes against the three elements of SPT, including adding in a code on the material element of sexting. This was crucial for analysing the normative regulations of sexting as a practice. Both authors coded the transcripts and compared codes across all stages of analysis.

\section{Applying practice theory to sexting}

This section is structured around the three main components of SPT, i.e., meanings, materials and competencies/skills. As the study was not designed around these three dimensions, we have limited data available on the materiality of sexting. We therefore begin with a brief analysis of this, before moving to the two main aspects of our analysis; meanings and skills.

\section{The materiality of sexting}

The materiality of sexting includes not only the prioritisation of the smartphone as the primary method for young people's sexting (rather than computers) (Van Ouytsel et al., 2017), but also relates to the various digital technologies and apps and their associated affordances (Hutchby, 2001). Participants generally indicated that various technologies and platforms (including Snapchat, Tinder, Whats'App, Facebook Messenger) are used differently, and indeed that the rise of such platforms has an impact on what sexting looks like. This is clear from the following quote, taking Snapchat as an example:

Warren: I think it's changed a lot, especially with the shit like Snapchat, where it's just temporary [...] people aren't as worried about sending that shit [highly sexualised visual content] now as they would have been beforehand. Especially because, [text] messages it's like, you can't really prove I sent that, but a photo it's clearly you. You know what I mean? You can't just be like, "oh yeah, my mate sent the text!" [...] If you send a photo then it's definitely you. [...] Guy: I think the technology that allows very easy image sharing now has changed the game in terms of sexting. Like five years ago it would be much more text based, like these examples [in the stimuli material], nowadays it's much more like visual and all that sort of stuff. 
The ephemeral dimension of Snapchat that Warren discusses is also considered by Handyside and Ringrose (2017). They argue that Snapchat "mediates how gender and sexuality are performed and negotiated and remembered' (Handyside and Ringrose, 2017: 11-12, emphasis in original) because of its temporary character. However, this is complicated by simple screenshots or other software that saves the 'snaps', thereby creating uncertainty about whether a snap was saved or only remembered. In the last part of the above quote, Guy explains the dominance of image-based sexting experiences amongst our participants with the technological developments that has made it easy and cheap to send such images. Sexting via phone texts was often described as more difficult and challenging across the sample, but also, as Warren notes, in some respects safer because it does not reveal the sender. Hence, different technologies clearly matter for how sexting unfolds and is experienced.

The material dimension of sexting also involves bodies, physical and mediated. Different bodies and body parts are assigned different value (e.g., 'fit' versus overweight bodies, breasts versus 'vagina shots') and can yield different affective responses (pleasure or disgust) when mediated (Authors XX). As we return to below, that sexting (typically) happens between people not physically together has implications for learning the practice.

\section{The meanings of sexting}

The purpose of sexting might be seen as leading up to sex. However, this assumption was complicated in the focus groups, as this exchange illustrates:

Greg: I think it [sexting] is any communication on a piece of technology that involves either writing or the sending of images of sexual content. Ziggy: Yeah, any intimacy in that way, that's what I think defines it.

Martin: I reckon there's always an endgame. I reckon... yeah, I mean, unless you get off with just hearing people and seeing what they're writing to you, I don't know. I think there'd have to be an endgame, because sex is obviously the top part.

Jerry: I think it also depends on the different types of endgame as well. There's obviously the endgame of getting turned on through pictures and stuff, but then there's also the endgame of building up this sexual relationship and building and building until you get to see the person and act upon what you'd typed kind of thing. 
While all groups discussed sexting as always having 'a purpose' or as Martin said above, "an endgame", the overarching meaning of sexting is multi-faceted and covers a range of 'purposes'. These range from instant sexual gratification to sustaining the momentum "consolidating a relationship", and, as Jim said in another group, to building up expectations to have sex. This is extended in the following:

Lyle: It depends who you sext with. If it's, like, with someone that you've known and that you're in a thing with, it's definitely like foreplay, it's leading up to sex. Harrison: I'd say it's usually about foreplay.

Lyle: But some people just enjoy it [for its own thing].

This is illustrative of participants' frequent suggestions that the meanings of sexting depend on who the receiver of the sexts is; whether this is a partner or a person one is flirting with. In that sense, sexting carries multiple meanings which creates the potential for misunderstandings. Importantly, though, sexting is clearly demarcated from sexual harassment or 'revenge porn' (cf. Henry et al., 2017), where the meanings are very different. We explore this further below, as our analysis turns to the men's perceived 'rules' of legitimate sexting.

\section{Defining appropriate sexting: mutuality \& consent}

In the data, sexting is woven into a discourse of mutuality and reciprocity, with clear contentions that both parties should be contributing to the practice for it to be sexting. It is also clear that 'good' sexting is characterised by a step-wise build-up, where each person's next step is a reaction to the interlocutor's previous message:

Quinn: I think it's often transactional. I honestly have not really ever, I don't think I've ever successfully sexted, but I think the times that I have briefly encountered it, it is transactional in the sense of, I'll give you this much, and they'll give you this much, but you give them $\mathrm{X}$, and they give you $\mathrm{X}$ plus one, and then you'll give them $\mathrm{X}$ plus two. It's kind of this, how much can you... I think that's where the mutuality comes into it, you both are getting a thrill out of, “oh, what are they going to do next? If I do this, what will I get back?" 
Leo: Yeah and that idea also of kind of, escalating mutual thing, I think probably that's an interesting one again on the line between flirting and sexting. Particularly with easy messaging apps like Snapchat now, where kind of like a sexy selfie, I wouldn't even think of as like a sext, but probably when you start to get past PG and into MA15 ${ }^{\text {iii }}$, that territory...

Quinn describes here a 'transactional' logic of sexting or 'the algebra of sexting, iv , where the intensity increases carefully and gradually. This was evident across numerous groups, with Wayne in group 4 noting that 'build-up is important, and being able to read where the other person is at'. We see in the extract above how 'the line' between appropriate and inappropriate sexting starts to take form in that sending "X plus 2" too soon would breech collective expectations around sexting; it would violate the norm of a slow build-up. Leo adds to this by using this 'escalating' logic to separate sexting from flirting and describes how flirting can turn into sexting when it moves into 'that territory' of explicit sexual content. Finally, the quote sheds light on the affective dimensions of sexting; on the one hand the positive aspects such as the 'thrill' but also the experience of waiting nervously and not knowing 'what will I get back?'. We go further into this insecurity below.

The emphasis on mutuality was mirrored in a strong discourse around consent and trust, with one group describing these as "of paramount importance". Across the data, consent was mentioned affirmatively 49 times (not counting 'consensual' and synonyms like respect, 'what they want' etc.). However, translating this into the sexting practice is complicated:

Simon: It [stimulus example] feels like consensual sexting.

Krish: Because it's coming from both ways.

Leon: They're both as explicit as each other.

Moderator: So what's the role of consent here? That's quite interesting, what do you mean by that?

Simon: So consent is really if they replicate...

Krish: [you mean] Reciprocate.

Leon: I feel like consent in a situation like this, wouldn't like be something that you'd necessarily communicate online. It would be more of an understanding of each other $[\ldots]$ 
Moderator: Is reciprocation and consent, whatever you want to call it, is that an important point?

Leon: Well yeah, because you know where the other person stands. Otherwise you could definitely say that it's harassment. I would genuinely class it as sexual harassment. I don't know if legally it is.

While consent is seen as key to sexting, the quote also illustrates that ensuring consent is tricky and not something that is explicated. Most focus groups reflected on this paradoxical situation noting that explicitly seeking consent would "ruin the vibe" (Jim, Group 6). Hence, consent must be sought more subtly, or as other groups put it, “it's more implied by both people's reactions" (Lyle, Group 10), “it's that you both understand what's going on" (Warren, Group 3), or "you both need to want the same thing" (Jacob, Group 4). Crucially, there is no face-to-face interaction in which such reactions can be read by body language; the only way of knowing these reactions is in terms of what is coming back. As Tim (Group 6) says: 'If you get a text back, like, "oh yeah, we're sexting”". This creates a basic uncertainty when engaging in sexting practices and as with the differences in specific meanings demonstrated above, this paves the way for misunderstandings and misread situations - and does to some extent questions the notion of a consensual practice in the first place. One way of reading this is that sexting is ideally a practice that is consensual and reciprocal; this is central in the meanings associated with sexting as being different from harassment, but in practice this ideal is hard to ensure.

\section{The (tacit) skills of sexting}

The vague definitions and blurred lines around sexting as a practice make some of our participants feel that sexting is sometimes a 'dangerous game' (Jacob, group 4). This is related not just to fears about whether one might breach codes of consent, implicit in the cultural meanings of sexting, but also to an underlying concern about whether one might appear 'pushy' or 'desperate'. However, this uncertainty does not preclude our participants from engaging in sexting practices, nor does it make them critical of sexting. Hence, appropriate sexting is a practice that must be learned.

Observing others is central when learning new practices. As Halkier writes, 'Practices ... come into being in the process of activities being regularly carried out in front of, together with and in relation to others' (Halkier, 2017: 397). Or as Warde elaborates, 'the novice is 
influenced by clues and cues about how other people, and particularly how other competent actors, navigate and traverse the sequences of actions that comprise recognisable performances of the Practice' (Warde, 2016: np). However, as Jackson \& Scott (2017) argue in their study of 'everyday sexualities', this is not the case with sex. Sexting, though, is different again as there is no face-to-face or embodied interaction to rely on; only the potential response from the other via digital platforms. This means that there is no 'learning by observation', only learning by doing. This is clear in the following quote:

Caleb: You never learn in a formal context. You never sit down with your mates and say, "how do I sext?" At all. You learn as it's happening. Moderator: How do you learn where someone's boundary is though? Scott: I feel like it's fairly easy to determine when someone's getting uncomfortable? Even if it is...

Kevin: I guess you just never try and reach that point. You never deliberately cross that line.

As Caleb says, learning to sext is learning "as it is happening". This means learning by engaging in sexting with a person one is sexually interested in - making the risk of 'failing' more serious. As the quote demonstrates, learning to sext is not only about learning the transactional logic described above, but also learning how to tell the receiver's boundaries, despite not being able to engage in face-to-face communication. As the young men here indicate, this is important because you do not want to "cross that line". It is also, however, something that is presented as almost common-sense and "fairly easy to determine". This suggests sexting practices, like other social practices, are "always influenced by the wider culture in which this learning occurs" (Jackson and Scott, 2017). This is also evident in participants' discussions about what kind of person does not 'cross the line':

Karl: I guess not so much a moral person, but someone that gets social cues, and knows when the line has been crossed, or when it's time to stop.

Matt: A solid grasp of social norms.

Tim: But if you're talking to someone [face to face] about something and you can tell they feel uncomfortable, you're not going to continue talking to them about that, so I suppose it's kind of the same thing as sexting.

Jim: A socially aware person. 
This response, and many like it, contradicts the uncertainty with which many of our participants engage in sexting practices. Co-existing with a distinct focus on the stepwise escalation and the complexities of ensuring consent, the participants state that knowledge about how to not cross the line is implicitly available to those who are 'socially aware'. This suggests the presence of tacit knowledge, derived from 'the learning of effective procedures, founded in much repeated experience, which mostly short-circuits processes of deliberation' (Warde, 2016: np). Sexting is thus a seen as a practice that is (partly) underpinned by embodied knowhow pertaining to its normalisation and wider acceptance, but which also constantly interacts with a wider range of implicit and central uncertainties.

This interaction of uncertainties, embodied knowhow and the relational other (the sexting recipient) is perhaps best evidenced through the participants' discussion of why they avoid 'crossing the line'. This was a strong preoccupation in most groups as is evident in the discussion below:

Moderator: .... but why would you stop? If you feel the other person is uncomfortable...

Matt: You don't want to be seen as weird.

Tim: You don't want to creep them out.

Liam: Well given that you're trying to get some sort of sexual connection with this person, you wouldn't want to compromise your chances further, by having them think that you're some massive creep.

Karl: Or compromise your chances with other people.

Liam: Yeah true, because they could pass on that information.

Here, we observe that transgressing the norms for good or acceptable sexting to the point of making the sext recipient uncomfortable is undesirable for multiple reasons. There is a concern for the other person, mirroring the sentiment in the quotes above about ensuring consent, seeking 'mutual engagement' and not wanting the other person to feel uncomfortable. But there is also a self-interest involved in this, as 'compromising your chances' would be to sacrifice one's chances of a mutually reciprocated sexual connection with this person, in the moment and in the future. Further, another risk with longer-term consequences is to be perceived as a 'creep' (see Author, XX), which leads to reputational 
damage and a subsequent diminishment of one's own sexual attractiveness to future possible partners. Accordingly, learning to become a 'good sexter' is not only related to concerns about the comfort of potential partners but, coming back to the meanings of sexting, also about being able to reach the 'endgame' of one's desire, whether that is some sort of immediate or delayed sexual gratification or the establishment of a longer term sexual relationship.

\section{Discussion: Theorising sexting as a social practice - gains and limitations}

Our analysis demonstrates sexting is a normalised, integral part of young people's wider sex lives and a practice that is seen as distinct from both flirting and harassment. It is, however, also a complicated practice. Even though the participants could easily identify 'appropriate' and 'inappropriate' sexting, this did not translate into confident engagements in sexting practices. What stood out in the analysis is the prominence of consent as central in the young men's understandings of appropriate sexting as well as the challenges in ensuring this. The emphasis on consent and mutuality, which sits in contrast to previous studies, might be a consequence of our participants' older age and assumed active sex lives. Nonetheless, this is an important and positive finding because it troubles the dominant negative representations in some previous research of young men. On the other hand, the challenges involved in obtaining consent mean that despite 'good intentions', there is much room for misunderstandings, insecurity and transgressing 'the line' in how sexting as a practice unfolds. This complicates the otherwise unambiguous distinctions that participants made between sexting and harassment/abuse, especially because instances where sender and receiver are not aligned in their exact understandings of the meanings of sexting are likely commonplace. The men's 'good intentions' around consent and mutuality are also somewhat relativised by the clear self-interest that plays into this motivation to engage in appropriate sexting practices.

These insights are enabled by approaching sexting as a social practice. Analysing cultural conventions and expectations along with actual performances is not necessarily very different from sociological understandings of sexual script theory. A practice theoretical approach, though, offers the added focus on how these cultural understandings and norms are translated into, first, competencies and then actual practices. This is also where we find a paradox in the data: sexting is at once normalised and embedded in participants' everyday (sexual/romantic) lives, but also perceived as something that requires mastery and tacit 
knowledges which make it a difficult and sometimes daunting endeavour. These complexities, and those detailed in the above paragraph, however fit well within the terrain of practice theory. For instance, the data we have offered here speak to Warde's (2016: np) contention that in articulating or displaying knowledge of practice, people 'divulge the normative dimension of the activity, which is circulating in public codes and discourses, and upon which individuals draw when required'. As we noted, though, the learning process involved in sexting is different from many other practices as sexting is learnt in an intimate, digital space in interaction with the person one is interested in - without any opportunity for observing others and no face-to-face interaction to help assess the other person's reactions. This is an important advance in SPT, extending the theory's hitherto emphasis on the necessary physical co-presence of other 'competent actors' (Warde, 2016) for learning a social practice. The practice of sexting is learned by doing, but also through the mediated responses one gets (or does not get) from an interlocutor. We contend that this is possible due to the strong shared meanings and cultural norms surrounding sexting; the clear emphasis on consent and mutuality.

Like all social theory, SPT has limitations. While beyond the scope of this paper to rehearse and address all these (see e.g. Schmidt, 2018; Warde, 2014) some are especially important when conceptualising sexting as a social practice. One limitation, or perhaps challenge, is methodological. Translating or operationalising SPT into empirical research carries multiple challenges (Halkier \& Jensen, 2011), in particular pertaining to the routinised nature of practices and the tacit and embodied skills. Having participants verbalise tacit aspects of naturalised and taken-for-granted practices is tricky, as we saw when asking participants how they know the boundaries regarding what to send in a sext. Our use of stimulus materials aided us by providing concrete examples of sexting that participants could discuss and other potential ways forward can be the use of creative methods that 'make the familiar strange' (Mannay, 2010).

Another, perhaps more important, limitation is analytical. Practice theory does not account well for social structures (Warde, 2014). In our case, this limits the analysis of the broader power dimensions and gender inequalities in which the practice of sexting is embedded. That is, sexting as a practice is framed by gender 'double standards' that have implications for the 'exchange value' of sexts as well as their gendered risks. This means young men and women face different sets of challenges when engaging in in sexting, but these are not brought to the 
fore with a practice-theoretical lens. However, they are crucial for a full understanding of sexting as a phenomenon. Accordingly, we have elsewhere supplemented our practicetheoretical analysis with an analysis drawing on a feminist conceptual framework geared towards this problematic (see Author, XX).

Our study also has some empirical limitations. We have, for instance, focused only on university students. We do not suggest that higher levels of education might mean more progressive attitudes - after all, highly problematic, misogynistic behaviours have been widely observed among educated men, in elite college fraternities for example (Seabrook \& Ward, 2018) - but we can only speculate about how our participants differ from, for instance, young men in the trades or retail sectors. Secondly, given our focus on young men's experiences and understandings of sexting, a parallel investigation with young women would more fully expand our understanding of sexting as a phenomenon in young people's lives. Comparing men's and women's experiences might also help identify gendered norms around sexting, going some way towards tackling the limitations described above (without accounting for the structural factors behind this).

Despite these limitations, SPT has numerous benefits for understanding sexting. It enables us, in the first place, to understand how sexting as a practice is facilitated by technological developments (and hence how sexting has come about). Furthermore, it allows us to situate sexting as a normalised albeit difficult practice in the everyday lives of young men, thereby adding to the growing literature on masculinity and sexual practices that problematises the focus on risk (Waling \& Pym, 2019; McCormack \& Wignall, 2017). Perhaps most importantly, SPT directs our analytical focus away from considering individuals as the sources of actions and instead towards the 'subtle dance between practices as individual performances and practices as embedded in a cultural structure' (Halkier et al., 2011: 9). As practices exist beyond the individual, practice theories point our attention to the social level; to the meanings associated with sexting as well as to the normative expectations surrounding it, rather than being a matter of (male) individuals making poor choices. The social level is also where to look in terms of understanding the positive as well as negative aspects of sexting and potentially intervening in harmful practices. 


\section{References}

Albury K, Crawford K, Byron P, \& Mathews B (2013) Young people and sexting in Australia: ethics, representation and the law. Sydney: UNSW.

Anastassiou A. (2017) Sexting and Young People: A Review of the Qualitative Literature, The Qualitative Report, 22(8):2231-2239.

Armstrong E, Hamilton L, \& England P (2010) Is hooking up bad for young women? Contexts, 9(3):22-27.

Barbour R (2007) Doing focus groups. London: Sage.

Bennett A (2004) Virtual Subculture? Youth, Identity and the Internet, in Bennett A and Kahn-Harris K (eds.) After Subculture, Hampshire: Palgrave Macmillan

Cohen S (1972) Folk evils and moral panics. London: MacGibbon \& Kee.

Demant J (2012) Natural Interactions in Artificial Situations: Focus Groups as an Active Social Experiment. In L Naidoo (Ed.), An Ethnography of Global Landscapes and Corridors. Rijeka: InTech.

Grogan S \& Richards H (2002) Body Image: Focus Groups with Boys and Men, Men and Masculinities, 4(3):219-232.

Halkier B, Katz-gerro T \& Martens L (2011) Applying practice theory to the study of consumption: Theoretical and methodological considerations, Journal of Consumer Culture, 11(1):3-13.

Halkier B \& Jensen I (2011) Methodological challenges in using practice theory in consumption research. Examples from a study on handling nutritional contestations of food consumption. Journal of Consumer Culture, 11(1):101-123.

Halkier B (2017) Practice Theoretically Inspired Focus Groups: Socially Recognizable Performativity?. In R Barbour, D Morgan, (eds) A New Era in Focus Group Research. Basingstoke: Palgrave. 389-410.

Handyside S \& Ringrose J (2017) Snapchat memory and youth digital sexual cultures: Mediated temporality, duration and affect. Journal of Gender Studies, 26(3):347-360. Harvey L \& Ringrose J (2015) Sexting, Ratings and (Mis)Recognition: Teen Boys Performing Classed and Racialized Masculinities in Digitally Networked Publics In: E 
Renold, Ringrose J and Egan, RD, eds. Children, Sexuality and Sexualization. Basingstoke: Palgrave. 352-367.

Hasinoff, A. A. (2014). Sexting as media production: Rethinking social media and sexuality, New Media and Society, 15(4):449-465.

Henry N, Powell A \& Flynn A (2017). Not Just 'revenge Pornography': Australians' Experiences of Image-based Abuse: Summary Report. Melbourne: RMIT University.

Hutchby I (2001) Technologies, Texts and Affordances. Sociology 35(2):441-56.

Jackson S \& Scott S (2017) Practice Theory and Interactionism: An Integrative Approach to the Sociology of Everyday Sexuality? In King, A. et al (eds.) Sexualities Research. London: Routledge. 70-82.

Klettke B, Hallford D \& Mellor D (2014) Sexting prevalence and correlates: A systematic literature review. Clinical Psychology Review, 34(1):44-53.

Kosenko K, Luurs G, Binder A (2017) Sexting and Sexual Behavior, 2011-2015: A Critical Review and Meta-Analysis of a Growing Literature; Journal of Computer-Mediated Communication, 22(3):141-160.

Lee M, Crofts T, McGovern A \& Milivojevic S (2015) Sexting among young people: Perceptions and practices. Trends \& issues in Crime and Criminal Justice, 508.

Madigan S, Ly A, Van Ouytsel, J \& Temple J (2018) Prevalence of multiple forms of sexting behavior among youth: A systematic review and meta-analysis. Pediatrics, 172: 327-335.

Mannay D (2010) Making the familiar strange: can visual research methods render the familiar setting more perceptible? Qualitative Research, 10(1):91-111.

Maller C (2015) Understanding health through social practices: performance and materiality in everyday life. Sociology of Health \& Illness, 37(1):52-66.

McCormack M \& Wignall L (2017) Enjoyment, exploration and education: Understanding the consumption of pornography among young men with non-exclusive sexual orientations. Sociology, 51(5): 975-991.

McDaniel B \& Drouin M (2015) Sexting among married couples: Who is doing it, and are they more satisfied?. Cyberpsychology, Behavior, and Social Networking, 18(11):628-634. 
Meyer S \& Lunnay B (2013) The application of abductive and retroductive inference for the design and analysis of theory-driven sociological research. Sociological Research Online, 18(1):1-11.

Mitchell KJ, Finkelhor D, Jones LM and Wolak J (2012) 'Prevalence and Characteristics of Youth Sexting: A National Study', Pediatrics 129: 13-20

Pini B (2005) Interviewing men: Gender and the collection and interpretation of qualitative data. Journal of Sociology, 41(2):201-216.

Reckwitz A (2002) Toward a Theory of Social Practices A Development in Culturalist Theorizing. European Journal of Social Theory, 5(2):243-263.

Ringrose J, Harvey L, Gill R, \& Livingstone S (2013) Teen girls, sexual double standards and 'sexting': Gendered value in digital image exchange. Feminist Theory, 14(3):305-323.

Ringrose J, Gill R, Livingstone S, \& Harvey L. (2012) A qualitative study of children, young people, and sexting. London: NSPCC.

Ringrose J \& Harvey L (2015) Boobs, back-off, six packs and bits: Mediated body parts, gendered reward, and sexual shame in teens' sexting images. Continuum, 29(2): 205-217. Salter M (2016) Privates in the online public: Sex (ting) and reputation on social media. New Media \& Society, 18(11): 2723-2739.

Salter M, Crofts T, \& Lee M (2013) Beyond criminalisation and responsibilisation: Sexting, gender and young people. Current Issues in Criminal Justice, 24(3): 301-316.

Schmidt K (2018) Practice theory. A critique. In: V. Wulf, et al. (eds): Socio-Informatics: A Practice-based Perspective on the Design and Use of IT Artifacts. Oxford: OUP. 105-137.

Seabrook R, \& Ward L (2018) Bros will be bros? The effect of fraternity membership on perceived culpability for sexual assault. Violence Against Women, Online first.

Shove E, Pantzar M, \& Watson M (2012) The Dynamics of Social Practice. London: SAGE. Swidler A (2001) What anchors cultural practices. In T. R. Schatzki, K. Knorr Cetina, \& E. von Savigny (Eds.), The practice turn in contemporary theory. Abingdon: Routledge. P239. Symons K, Ponnet K, Walrave M, \& Heirman W (2018). Sexting scripts in adolescent relationships: Is sexting becoming the norm? New Media \& Society, 20(10):3836-3857. 
Törrönen J (2002) Semiotic theory on qualitative interviewing using stimulus texts.

Qualitative Research, 2(3):343-362.

Van Ouytsel J, Walrave M, Ponnet K (2018) A Nuanced Account: Why Do Individuals

Engage in Sexting?. In: Walrave M., Van Ouytsel J., Ponnet K., Temple J. (eds) Sexting.

Palgrave Studies in Cyberpsychology. Basingstoke: Palgrave Macmillan.

Yadlosky L, St. Aubin E, Mosack K and Devine J (2017) 'Sexual Perceptions Versus Reality in Undergraduates: Data-Driven Praxis for Sex-Related Campus Programming' Journal of College Student Development, 58(5):704-718.

Walrave M, Van Ouytsel J, Ponnet K, Temple J (2018) Sharing and Caring? The Role of Social Media and Privacy in Sexting Behaviour. In: Walrave M, Van Ouytsel J, Ponnet K, Temple J. (eds) Sexting. Palgrave Studies in Cyberpsychology. Basingstoke: Palgrave Macmillan.

Waling A \& Pym T (2019) 'C'mon, No One Wants a Dick Pic': exploring the cultural framings of the 'Dick Pic'in contemporary online publics. Journal of Gender Studies, 28(1): 70-85.

Warde A (2016) The practice of eating. London: John Wiley \& Sons.

Warde A (2014) After taste: Culture, consumption and theories of practice. Journal of Consumer Culture, 14(3):279-303.

\footnotetext{
i Image-based abuse, a term adopted by Australia's e-safety commissioner, refers to intimate, nude or sexual images being distributed without the consent of those pictured.

${ }^{i i}$ For copyright reasons the stimulus materials cannot be reproduced here, but examples of the materials are included in the online appendix.

iii This refers to Australian film classifications. PG classified films/games require parental guidance for children under age 15 and are 'mild in impact'. Films classified as MA15+ are for people aged 15 and over as 'the content is strong in impact', i.e., may contain sex scenes and drug use. http://www.classification.gov.au/Guidelines/Pages/MA15+.aspx

iv This term was coined at the XX conference by YY. We thank him for this contribution.
} 


\section{University Library}

\section{- M M N E R VA A gateway to Melbourne's research publications}

Minerva Access is the Institutional Repository of The University of Melbourne

Author/s:

Roberts, S;Ravn, S

Title:

Towards a Sociological Understanding of Sexting as a Social Practice: A Case Study of University Undergraduate Men

Date:

2020

Citation:

Roberts, S. \& Ravn, S. (2020). Towards a Sociological Understanding of Sexting as a Social Practice: A Case Study of University Undergraduate Men. Sociology: the journal of the British Sociological Association, 54 (2), pp.258-274. https:// doi.org/10.1177/0038038519864239.

Persistent Link:

http://hdl.handle.net/11343/294913 